\title{
Formation, Concept Development in Pedagogical Environment and Educational Solutions to Improve Conceptualization for Vietnamese Students
}

\author{
Dr. Tran Thi Ngoc Anh \\ Hanoi National University of Education, Viet Nam
}

\begin{abstract}
Concepts are both the result of the reflection of the human objective world, and the reasoning means for people to continue to perceive and improve the world. Concept is the first stage in the process of perceiving human reason. Comprehending the concept, in order to be effective, requires a lot of effort and effort of the subject of awareness, needs to have a certain understanding of the law of perception, the law of psychophysiology ... and the help of people. ahead.

Keywords: Concept formation, Concept development, pedagogical environment, Comprehension improvement solution, Concept.

DOI: $10.7176 / \mathrm{JEP} / 12-10-06$

Publication date: April $30^{\text {th }} 2021$

\section{Preamble}

In practical activities, realizing the correct reality to transform towards the benefit of people is a requirement that always comes first. This cognitive process is a dialectic process through many stages, in which concept is an important suture. Currently, reality has profound changes at a rapid rate, which is the condition for a series of new scientific concepts to be born. Therefore, we need to master, develop and apply these new concepts flexibly in the innovation process, especially in the current conditions of a knowledge economy.
\end{abstract}

\section{Research content}

\subsection{The formation and development of concepts in pedagogical environment}

This is an issue that has been of concern in the education world for many years. In 1950, with a target of middle school students, A.Z.Retô (Former Soviet Union) investigated "The comprehension of historical concepts by fifth and seventh grade students". At the 1955 Conference on Psychology in Moscow, R.T. Natazea presented a report "on grasping specific, natural science concepts in high school". In 1956, NFTaluzina studied "The perception of the intrinsic signs of concepts in organized action of the experimental object." Then, in 1959, the AMGondobec did an experiment with the object of middle school students in Ukraine to learn "A two-way way of acquiring common scientific concepts of young students".

In Vietnam, although there are not many studies in this field, but educators are always interested in how to help the process of forming concepts for students better. Right from the war years (1964-1965), Pham Hoang Gia and the thinking research team of Hanoi Pedagogical University have investigated in some Northern localities about the 6th and 7th grade students Assembly concepts math, literature, biology like. By 1979, some results were also stated in his thesis "The nature of intelligence and theoretical basis of the way of conceptual comprehension".

At the Institute of Educational Science, from 1974 to 1980, the Psychology and Teaching group chaired by $\mathrm{Ha} \mathrm{Vi}$ studied the formation and conceptualization of elementary school students, from which the group drew the results. Discussing the mechanism and laws of this process, proposing the re-structuring of lectures and teaching hours, turning material activities into spiritual activities for the children. In each specific science, finding positive solutions to help students master concepts, there are also a number of theses and theses, for example by the authors: Nguyen Ngoc Quang with the formation of concepts. chemistry in high school (1970), Tran Ba Hoanh with biological concepts in grades 9 and 10 (1975), Cao Gia Nuc with concepts of "ecology" in botanical programs, Pham Trung Thanh with math concepts level I (1983), Nguyen Giang Tien with geography concepts in grades 10 and 11 (1985), Nguyen Hieu Trien with concept of "fractional" for students in grades 4 and 5 (1991) ... At university level, there are the authors: Do Hanh Phuc with the concept of psychology in students of math, culture, and culture faculties of Hanoi Pedagogical University (1980), Nguyen Huu Long with mental concepts. physics for pedagogical students (1987) ...

In general, the topics all exploit the formation and development of concepts for students of a particular science, and the subjects are students of a certain level, most of them are high school students under the corner. educational science degree. These are extremely valuable suggestions for us to approach this issue.

Pedagogical environment is understood as a special environment, with a unified goal, in which a long-term educational process is conducted, which is essentially a process of transferring and receiving concepts from 
generation to generation. in which the subjects have a mutual interaction relationship. Concept formation and development in pedagogical environment always give themselves two parallel tasks: one is equipping knowledge with the existing conceptual system, the second is turning them into skills, skills, and abilities. practices for the perceived to develop their intellect. The philosophical basis of that phenomenon is the determination and resolution of a contradiction in the cognitive process of the subject, the contradiction between self-control and controllability of the learner's cognitive system. This conflict is one of the driving forces behind cognitive development.

\subsection{Regarding the curriculum structure, teaching facilities and the subject of transfer of concepts}

The basic relationship of the process of concept formation and development in the pedagogical environment is the interaction between two subjects: the subject transferring the concept (the teacher, the teacher) and the subject perceiving the concept. (student). In order to solve this relationship well, the knowledge program is put into textbooks, learning materials, textbooks ... in addition to exact and scientific requirements, it must be distributed to maximize the proactivity., creativity of the subject.

The curriculum needs to ensure a close relationship between grades, grades and between subjects. Pedagogical environment must be understood in the sense of a unified pedagogical space, in which the process of transferring and receiving concepts in a long way, without being fragmented. It is not unreasonable that the same concept can be encountered in many different grades and subjects at different levels and approaches. The concept of "whole number", for example, was introduced to first graders by first graders, but the concept is still coming back into the curriculum of the following grades, becoming more and more difficult. more statues. Or as the concept of "molecule", which can be found in chemistry, physics, in each block, each subject has its own specificity. In addition, the priority of prerequisite subjects, basic concepts, that if not equipped, learners will be very confused when receiving the next concepts, even unable to receive.

The basic theory and practice part of learners should have an appropriate ratio for each subject, each lesson, especially with subjects of social sciences. Until now, the natural sciences were easier to divide these two parts, helping to convey and absorb concretely concepts, and for concepts in the field of social sciences, it was very difficult. such division, because they are both highly abstract, the program structure is heavy in theory.

The program structure has a choice to suit each object, deriving from scientific requirements and ensuring practicality. The public opinion is very interested in the reform of education today, and there are many opinions that reforming textbooks, although very necessary, has many shortcomings, because the program content is not suitable in many places. objects and requirements of practice. This reform happened lack of synchronization between grade levels and levels, it can be said that it is fragmented, to be followed in time. Whether doing like this reduces the quality of teaching and learning in that grade, and in turn affects other grades, this question has received a lot of feedback. In our opinion, education reform is essential to fit the new conditions, but it needs to be carefully prepared so as not to break the logic of the education process in general.

About teaching facilities, including media such as writing materials, textbooks, reference books, visual aids, and modern teaching facilities such as computers, videos, projectors ... We do not abuse these media, but must know how to use them effectively, selectively combine, to support the teaching of concepts. Currently, the time spent on students and students in lecture halls should be shorter so that they have more time to self-study with support facilities, so that they become familiar with the way of self-training, choosing from many resources and also for them to train their own scientific thinking. For students of lower levels, the means of learning help them a lot in getting used to new concepts, especially abstract concepts. When watching live traffic law violation images of peers that lead to serious accidents via video, junior high school students can become more familiar with the concepts: " the law", "civic duty "... or the practice of connecting an electrical network by themselves, making a solution on their own makes it much easier for them to understand than to describe the experiment verbally.

Regarding the subject of transferring the concept (the teacher), they can be compared as a guide for students to go to new areas of knowledge. The requirements for them are very high: - Must have quite a wide background of knowledge, not only in one specialized subject but also in other subjects, especially the subjects that are related to each other. - Deep professional knowledge, a thorough understanding of the concept that needs to be conveyed: the content, the formation and development, the dependent factors, the similar or contrasting concepts, placing the concept in the program, in the system, forecast the development trend of the concept in the future ... Solid pedagogical profession, the way of transmitting scientific concepts, accurate, attractive, mainly organizing lectures or guiding learners conceptual approach, not cramming, heavy on conceptual explanation. Only then can promote the proactive nature of the perceiver. - Always self-improve comprehensively in order not to become out of date compared to reality.

The above operations in fact do not have a clear division that they can be intertwined and linked with each other. For example, the two operations of analysis and synthesis, many people believe that they are contradictory, but from a Marxist epistemological point of view "the method of philosophy is both synthesis and analysis, but is 
determined not to follow. that is, both of those methods of finite perception lie next to each other or are simply contiguous; it is that both are contained in the philosophical method in a stripped-down form, and in every step of it, the philosophical method acts both analytically and synthesically ". Analysis and synthesis, are not contradictory manipulations of thinking. "It seems that at first we, thoughtlessly, shred the bodies, and then try to collect and assemble it from those shredded parts. Such "analyzing" and "synthesizing" is more like a baby breaking a toy without hoping to "make it the same" than to the theorist. In fact, 'theoretical analysis from the outset takes place with care not to ruin the relations between the individual elements of the polity being studied, which, indeed, the contrary, is to clearly, to keep track of them, the reckless analysis (which loses the model of the body as a premise for his purpose) always has to take the risk of chopping up the object into components. into constituent parts, which for it are completely nonspecific and out of them, so to re-collect the whole is neither possible nor impossible when cutting the body into lumps and then reassembling the bodies. That Department brought the body back to life ".

Human perception of concept also depends on general psychological factors as well as of each specific individual. The teacher should know how to stimulate these factors in a positive direction.

Learning attitude is a necessary condition for conceptual understanding. Attitude can be only temporary, short-lived, can also become solid, dominating the learning process if properly nurtured. This is the duty of a good instructor, turning a positive attitude into a solid factor in the student's personality. Learning attitude is shown through attention and interest in learning. Attention is the essential condition for the success of conceptual understanding. "Attention is the door through which all the things of the outside world enter the human soul". Attention makes the mind disciplined, organized, creates a positive state. Experienced teachers always know how to control the student's attention, know how to promote the qualities of attention in specific situations. From the promotion of attention, learners will have cognitive interest, and conversely when cognitive interest appears, the more attention will be paid to the purpose of research and learning. Cognitive pleasure is the individual's selective orientation to a certain cognitive goal. "A perception without interest and only know how to act with forced force will kill the desire to learn in the individual."

Conceptual comprehension also includes memorizing, preserving collected information and can be retrieved at any time needed to use in new cases and circumstances. It is the ability to apply knowledge creatively - an indispensable criterion for assessing conceptual comprehension. Many people believe that conceptual comprehension only needs to stop at the level of understanding and remembering the essential signs and belittling this practical standard. Therefore, someone has proposed the algôrite solution for the rixtix path. Original algit is a mathematical concept used to calculate the regulation, precision and unit cost of completing thinking manipulations in a certain sequence in order to solve problems of a certain type or type. The algôrites are characterized by three basic properties: determinism (unvalue), series calculation (can be applied to solve a wide range of problems of the same type), calculation of results (if the correct procedure is guaranteed. manually manipulating, will definitely solve the correct problem given). In fact, the algôt solution is a formulaic solution that does not require much investment or thought. Before familiar situations, people seek answers by way of algôrít, but in new situations, people cannot only rely on existing formulas but must find answers through the rixtic way. This solution requires subjects to apply their intellectual capital creatively, actively seek and discover to solve problematic situations. New concepts need to be perceived and put before the subject as problem situations, are intellectual obstacles that stimulate the subject to solve. And the subject will not solve this task in a groping, undirected way, but according to a solution chosen for the problem situation is the most optimal. That is the algorite for the rixtic road. Schools and teachers cannot have the full ambition for students to solve every problem they will encounter in life, nor can they equip them with all the scientific concepts. And as analyzed before, the introduction, even thorough explanation of all concepts in the program is not the essence of the conceptual comprehension process. The role of the instruction (of teachers, learning materials ...) is to organize, direct, and outlines methods to help students self-explore to come to concepts. This is the basis for the policy of turning the training process into a self-training process, promoting students' self-awareness, initiative, creativity, and positive awareness. The teacher is not only a person with extensive knowledge, but also an expert in methods.

\subsection{Several educational solutions to improve comprehension of concepts for Vietnamese students 2.3.1. Regarding the curriculum structure, teaching facilities and the subject of transfer of concepts}

The basic relationship of the process of concept formation and development in the pedagogical environment, as analyzed in the first period, is the interaction between two subjects: concept transfer subject (teacher) and conceptual perceived subject (student, student). In order to solve this relationship well, the knowledge program is put into textbooks, learning materials, textbooks ... in addition to exact and scientific requirements, it must be distributed to maximize the proactivity., creativity of the subject.

The curriculum needs to ensure a close relationship between grades, grades and between subjects. It is not unreasonable that the same concept can be encountered in many different grades and subjects at different levels 
and approaches.

The basic theory and practice part of learners should have an appropriate proportion for each subject, each lesson, especially for social sciences subjects.

The program structure has a choice to suit each object, deriving from scientific requirements and ensuring practicality. The public opinion is very interested in the reform of education today, and there are many opinions that reforming textbooks, although very necessary, has many shortcomings, because the program content is not suitable in many places. objects and requirements of practice.

About teaching facilities, including media such as writing materials, textbooks, reference books, visual aids, and modern teaching facilities such as computers, videos, projectors ... We do not abuse these media, but must know how to use them effectively, selectively combine, to support the teaching of concepts.

Regarding the subject of transferring the concept (the teacher), they can be compared as a guide for students to go to new areas of knowledge. The requirements for them are very high: have a wide background of knowledge, not only in one specialized subject but also in other subjects, especially the subjects that are related to each other; Deep professional knowledge, a thorough understanding of the concept that needs to be conveyed; sure pedagogical profession, how to convey scientific concepts, accurately, and attractively, mainly organize lectures or guide learners to approach concepts rather than cramming, heavy on conceptual explanation; always self-improvement of comprehensive knowledge so as not to become out of date compared to reality.

\subsubsection{Want to perceive a concept, it is necessary to put that concept in the unity between logic and history}

The concept is formed from the requirement of reality, so when wanting to perceive a concept, the subject needs to examine the source of socio-political - social conditions that produce that concept. These conditions can also cause the connotation of a concept to change partially or completely, so in many cases the term has not changed but the concept has changed significantly. For example, when comprehending the concept of "worker" in Vietnam today, the perceiving subject must investigate the change of some socio-economic forms, from feudal society to capitalist society. to socialist socialism, the same socioeconomic form in other countries must be compared with Vietnam to find out specific socio-economic conditions. In the eleventh century, the premises for the concept of "worker" appeared in Vietnam. The development of feudalism, the need to build temples into citadels, pagodas, and a deep division of labor ... created a class of salaried people. People take the word "work" as the essence and then append words referring to the labor partner or the scope of labor to create a name for them. For example: in August of Tan Mui year (1013) "Refunds to pay for the money to create a private garden in the village of pham phat five decades" (Ly king pays workers to build 150 pagodas in the hamlets) (remuneration - hiring workers). In November of the year of the Mau tuat (1118), heather (stone worker) engraved eight words written by the king himself into the stele "Thien Ha Thai Binh". In the Tran dynasty, the Department of Polytechnic of the court had hundreds of workers working under the "statue" regime. In the Le dynasty, in the fifteenth century, there was a concept of "the late worker" to call the servants in families: "there are two types of servants in the families, the slaves and the late workers" [77]. In our folklore, we have the phrase "scholar, farmer, meritorious service" to refer to those who do some basic jobs. Labor for the state is called with the form of forced labor, often associated with the word "spouse": labor force, husbandry - labor in mining, nail - shipbuilding labor, chauffeur. - Labor exploiting and cooking zinc ... At the end of the nineteenth century, the French colonialists promoted the exploitation of colonialism, Vietnamese bourgeois and overseas Chinese bourgeois began to appear, develop production and business activities, thus Employees are used more (estimated at nearly 100,000 people), often called boat workers, starting to be modern (workers in mechanics, electricity, shipbuilding, mining ....). The above concepts are considered predictive concepts of the concept of "worker", through Vietnamese historical practices that are gradually formed. The concept of "worker" was officially born in the early twentieth century, when the industry began in the modern sense. But it was not until the $20 \mathrm{~s}$ of the twentieth century, when Marxism-Leninism was propagated into Vietnam that the concept of "worker" became a scientific concept in our country and was widely used. In the works of Marx, Angghen, the concept of "worker" and the concept of "proletariat" have the same connotation. In the "Declaration of the Communist Party", the two men wrote: "The proletariat are the modern working class, and because they lost their production materials, they were forced to sell their labor force. to live". They were the offspring of modern industrial production, but it was the industrial revolution that pushed them into proletariat, "the machines that gave the whole industry into the hands of the great capitalists and completely did it. reduce the value of the insignificant small assets belonging to the workers (tools, looms ...) so that soon, the capitalists have got everything in their hands, and the workers have nothing more "[88,1,442]. When Marxism-Leninism was introduced into Vietnam, in the early part of the 20th century, the Vietnamese working class were also proletariat. From the date of national independence, the working class together with the masses of people to own the country, the workers are no longer proletariat, and from 1955 onwards, the concept of "the Vietnamese proletariat" does not exists but instead is the concept of "modern working class Vietnam". There is an opinion that: "If based on the standards and characteristics that Marxist philosophy has raised, Vietnam still does not have a modern working class, for the reasons: Vietnam does not have capitalism. development, no great industry, so there is no 
premise for the emergence of the working class; This class must be the class of those with knowledge, high cultural and professional qualifications.

If this is understood as non-historical, because, over time, new conditions and factors will appear, old conditions and factors disappear, in each country, every continent has its own characteristics. separate points. Currently, the working class has also divided into manual workers, scientific research workers and service workers. This differentiation is required by specified practice, and in perception it is reflected in the respective concepts. The working class must now self-cultivate knowledge to meet the needs of the era. They continue to carry out their world history mission, but in a different social situation from the years in the last two centuries. The bourgeois and proletariat contradictions have also distorted, leading to the ever more complicated struggle between these two classes. To properly understand the current concept of "worker", there is no other way but to find and compare those "other social situations".

\subsubsection{To perceive a concept, it is necessary to find the unified content of the opposites reflected in that concept}

The concept reflects the nature of things and phenomena, as well as reflecting the internal contradiction within the phenomenal things. The person who understands the concept is the person who is able to analyze, find the basic contradictions of the things hidden in the connotation of the concept. For example, when finding out the concept of "fit" in the law of production relations, it must be consistent with the nature and level of the production force, the perceiving subject needs to see the duality of this phenomenon. According to the Vietnamese dictionary, the match is the match, the match is to match. The Pinyin-Vietnamese dictionary of Dao Duy Anh explains: fit is the bamboo tag used to make news, match is match, is the right match. In the past, people who had to be separated from each other, it was customary to take a bamboo card to write words on and then break them in half, each one piece, then bring them together to make news. Marx used the word ENTSPRECHEN (German) to indicate a necessary state of production relations so that the production force has conditions to develop, which can be translated into Vietnamese as appropriate, appropriate, adaptive ... In the article "The dialectic of production forces and relations of production" (Journal of Philosophical Problems, No. 5 , 1987], IU.K.Pletnhicop writes: "Correlation manifests itself by cohesion., identity in a certain extent, often understood as conformity. As a philosophical concept, conformity manifests itself as unity (identity), it does not annihilate but also contains contradiction. dialectical contradiction. "In general," suitable "in this case is a passive difference, in which there is an element of nonconformity. A wants to be called conformable B when A fully satisfies the word requirements. side B. Thus, a production relationship is said to be consistent with its respective production force, if the production relationship meets the requirements set by the production force in a certain period of time. If it is no longer suitable, it will be replaced by a more suitable production relationship.

\subsubsection{To receive a new concept, the subject must equip himself with a basic amount of knowledge}

Want to perceive a certain concept, the subject also needs a certain degree, a certain knowledge of things, phenomena that the concept reflects, the history of the problem (has been started and How far is it being studied?), there are the theories or the schools to study, how is it interested now and the research trend in the future ... A concept is formed. always is the result of the whole research process because the concept squeezes in the heart "the whole power of that content". To comprehend the concept, learners need a level equivalent to that of the concept, the level to a level of knowledge mature enough to be able to understand what the concept refers to. Reaching this level can be in many ways: practical activities to draw experience, collect relevant materials into a system to learn from human experience ... When criticizing natural scientists. Falling into metaphysics, Angghen emphasized the role of research into that treasure of knowledge, especially historical and philosophical knowledge:

"Whatever the natural scientists do, they are still dominated by philosophy. The only problem is that they want to be dominated by a trendy bad philosophy, or they want to be guided by a form of reasoning based on an understanding of the history of thought and its achievements. "The study of the Angghen" electric "concept is a case in point. In general, he affirms: electric phenomena are everywhere, "almost there is no change on the earth that people do not see the presence of electrical phenomena". However, research on it, until that time, has not found its clear nature. "In the theory of electricity we see a mess of old, uncertain, completely unconfirmed, and completely undetermined, groping, a series of fragmentary studies and experiments of many individual scientists are attacking a dispersedly new field, like a swarm of nomadic cavalry. To prove this, Angghen followed the electrical doctrines of Pharaoh, Thomas, Thomas ... individual. Followed by Peter and Vebov's views on electric current. These two men were not satisfied with the idea that in an electric current, either positively or negatively, passing through, they both asserted that in a closed current there are both equal positive and negative currents passing through. at the same time, one thread, beside the other, runs in opposite directions in the intermolecular pathways with the weight of the objects. Contrary to this theory, in 1871 , Noiman hypothesized that only one of the two currents, such as the positive charge, moves in the current, while the other, the negative electricity, is closely tied to the mass of the object. However, this hypothesis is also not stable because of the lack of grounds. With the experiments of Joseph, Peter, and Raun, electrodynamics was shown to be equal to the energy produced 
in the chemical processes of the electric cell, or by the energy dissipated in the electrolytic vessel. By this conclusion, it is assumed that electricity as a special liquid is more unstable. At that time, new theories of Cléc Maxuen (1864), Hanken (1865), Râyna (1870), Étlun (1872) all agreed with Pharaoh's earlier 1846 suggestion: electricity is a movement. of an elastic medium that pervades the entire space, thus permeating every object, a medium in which small dispersed particles push each other inversely proportional to the square of the distance; in other words, electricity is the movement of small etheric particles, and molecules of objects also participate in the movement. After that, Angene analyzed very meticulously every single theory of this movement, such as Vidơman's theory, contact theory of Amnta, old chemical theory ... He also described in detail each experiment, closely watched. Scientists' debates to come to a methodological conclusion: "And in fact, there is no doubt that wanting to give the theory of electricity ... a solid basis, there is only one way. is a correct revision ... all old experiments ..., with the condition of carefully observing and validating the energy transformations and all old theoretical perspectives set aside. power transmission ". Examine the entire history of the problem - it's also the way of researching to comprehend concepts in general.

\section{Conclusion}

\section{Wanting to perceive a concept, the subject needs to master the manipulation of thinking}

These are operations such as comparing, analyzing, abstracting, synthesizing, generalizing ... "The formation of concepts is not a direct transition from sensory perception results, on the contrary, on basis of emotional documents, in order to build concepts, we have been through a process of active and creative activities ".

The comparison operation helps subjects determine the differences and similarities between objects, from which they can be arranged according to defined groups, on that basis, determine the object class to be aware of in a multitude of objects. Phenomenal thing. Analytical operations require the subject to find the structure of the object, to study each element of the structure. With the result of the analysis process, the subject proceeds to abstract step, removing non-basic attributes and relationships, retaining only the basic relationships, it depends on the level of real activity. see and develop the subject's knowledge. After finding out basic relationships, the cognitive subject must conduct a synthesis operation in order to place properties and relationships in a unified body to have a consistent thought about the object. Analysis and synthesis, are not contradictory manipulations of thinking. "It seems that at first we, thoughtlessly, shred the bodies, and then try to collect and assemble it from those shredded parts. Such "analyzing" and "synthesizing" is more like a baby breaking a toy without hoping to "make it the same" than to the theorist. In fact, 'theoretical analysis from the outset takes place with care not to ruin relations between the individual elements of the polity being studied, which, indeed, the contrary, is to clarifying, to keep track of them, there is reckless analysis (which loses the model of the body as a premise for his purpose). it is always reckless to decompile the object into constituent parts, which for it are completely nonspecific and out of them, so to redraw the whole is not possible, just as well. It is impossible when cutting the body into lumps and then attaching them to bring the body back to life. The author's impressive comparison has caused many researchers to startled to see if they had fallen into such a dangerous mistake.

The process of coming to the concept is not finished if the subject has not done the generalization, in order to apply the results of the research synthesis to broaden the subject to arrive at the final, comprehensive and indepth conclusion about the subject. object, there is a complete concept of the object.

\section{Want to perceive a concept, the subject needs to promote positive psychological factors}

Learning attitude is a necessary condition for conceptual understanding. Attitudes can be temporary and short-lived, and can also become firmly dominant in the learning process if properly nurtured. This is the duty of a good instructor, turning a positive attitude into a solid factor in the student's personality.

Learning attitude is shown through attention and interest in learning. Attention is the essential condition for the success of conceptual understanding. "Attention is the door through which all the things of the outside world enter the human soul". Cognitive pleasure is the individual's selective orientation to a certain cognitive goal. "A perception without interest and only know how to act with forced force will kill the desire to learn in the individual." Conceptual comprehension also includes memorizing, preserving collected information and can be retrieved at any time needed to use in new cases and circumstances. It is the ability to apply knowledge creatively - an indispensable criterion for assessing conceptual comprehension. Many people believe that conceptual comprehension only needs to stop at the level of understanding and remembering the essential signs and belittling this practical standard. Therefore, someone has proposed the algôrite solution for the rixtix path. Original algit is a mathematical concept used to calculate the regulation, precision and unit cost of completing thinking manipulations in a certain sequence in order to solve problems of a certain type or type. The algôrites are characterized by three basic properties: determinism (unvalue), series calculation (can be applied to solve a wide range of problems of the same type), calculation of results (if the correct procedure is guaranteed. manually manipulating, will definitely solve the correct problem given). Before In familiar situations, people seek answers by algôt, but before new situations, people cannot only rely on the existing formulas but must find answers through the rixtic way. This solution requires subjects to apply their intellectual capital creatively, actively seek 
and discover to solve problematic situations. And the subject will not solve this task in a groping, undirected way, but according to a solution chosen for the problem situation is the most optimal. That is the algorite for the rixtic road. After that, a Russian educator, B.I. Kôchiaep, noted: "And the following rule is equally important: student learning outcomes will coincide with what is expected when and only if the purpose, content, forms and forms Teaching methods are completely commensurate with each other".

The above mentioned measures are just one of many measures to help form and develop concepts in an effective pedagogical environment. Subjects perceive should know how to take advantage of the favorable factors in the pedagogical environment, combine all of the above measures, and at the same time, with creative cognitive nature, they should also apply more other measures. more by themselves discovered on their educational path.

\section{REFERENCES}

1. Ho Ngoc Dai (1983), Teaching Psychology, Education Publishing House, Hanoi.

2. Le Van Hong (1994), Pedagogical Psychology, Hanoi University of Education I.

3. Nguyen Van Hoi (1992), Developing the optimal plan to control the cognitive process of students, Ph.D. thesis in philosophy.

4. Mark C., Angels Ph. (1994), Complete Works, (20), National Political Publishing House, Hanoi

5. Van Tao (1995), Historical method and logical method, Vietnam History Institute, Hanoi.

6. Vu Van Vien (1998), "The formation and development of concepts", Philosophy, (6), 31-35. 9.

7. Patrick J. Hurley (1991), A concise introduction to logic, Wadsworth Publishing Company, Belmont, California. 